\title{
The Effects of Taekwondo Training on Brain Connectivity and Body Intelligence
}

\author{
Young Jae Kim¹, Eun Joo Cha², Sun Mi Kim³ , Kyung Doo Kang ${ }^{4}$, and Doug Hyun $\mathrm{Han}^{3} \bowtie$ \\ ${ }^{1}$ Department of Physical Education, Chung-Ang University, Seoul, Republic of Korea \\ ${ }^{2}$ Physical Education LAB, Chung-Ang University, Seoul, Republic of Korea \\ ${ }^{3}$ Department of Psychiatry, Chung-Ang University Hospital, Seoul, Republic of Korea \\ ${ }^{4}$ Department of Exercise and Sport Science, University of Utah, UT, USA
}

Objective Many studies have reported that Taekwondo training could improve body perception, control and brain activity, as assessed with an electroencephalogram. This study aimed to assess body intelligence and brain connectivity in children with Taekwondo training as compared to children without Taekwondo training.

Methods Fifteen children with Taekwondo training (TKD) and 13 age- and sex-matched children who had no previous experience of Taekwondo training (controls) were recruited. Body intelligence, clinical characteristics and brain connectivity in all children were assessed with the Body Intelligence Scale (BIS), self-report, and resting state functional magnetic resonance imaging.

Results The mean BIS score in the TKD group was higher than that in the control group. The TKD group showed increased low-frequency fluctuations in the right frontal precentral gyrus and the right parietal precuneus, compared to the control group. The TKD group showed positive cerebellum vermis (lobe VII) seed to the right frontal, left frontal, and left parietal lobe. The control group showed positive cerebellum seed to the left frontal, parietal, and occipital cortex. Relative to the control group, the TKD group showed increased functional connectivity from cerebellum seed to the right inferior frontal gyrus.

Conclusion To the best of our knowledge, this is the first study to assess the effect of Taekwondo training on brain connectivity in children. Taekwondo training improved body intelligence and brain connectivity from the cerebellum to the parietal and frontal cortex.

Psychiatry Investig 2015;12(3):335-340

Key Words Taekwondo, Body intelligence, Cerebellum, Brain connectivity, fRMI.

\section{INTRODUCTION}

\section{Body perception and brain in sports}

The perception of body image is defined as the perception of one's body size, appearance, and boundaries. ${ }^{1}$ In addition, body intelligence is defined as awareness and body sensation originated from within the body and the body acting in response to other action or environments. ${ }^{2}$ Body perception consists of afferent multisensory information from outside and existing multifaceted neural body representation. ${ }^{3}$ Additionally, body representation is up-graded through a bidirection-

Received: August 30, 2014 Revised: November 11, 2014 Accepted: November 24, 2014 Available online: July 6, 2015

$\triangle$ Correspondence: Doug Hyun Han, MD, PhD

Department of Psychiatry, Chung-Ang University Hospital, 102 Heukseok-ro, Dongjak-gu, Seoul 156-755, Republic of Korea

Tel: +82-2-6299-3132, Fax: +82-2-6299-1508, E-mail: hduk@yahoo.com

(a) This is an Open Access article distributed under the terms of the Creative Commons Attribution Non-Commercial License (http://creativecommons.org/licenses/by$\mathrm{nc} / 3.0$ ) which permits unrestricted non-commercial use, distribution, and reproduction in any medium, provided the original work is properly cited. al calibration of process using body perception and past body representation. ${ }^{4}$ Proprioception is defined as an individual's sense of the current status of the parts of one's own body in space. It is mainly informed from afferent signals linked with muscle spindles, tendons, skin, and joints. ${ }^{4}$ Within human developmental stages, body perception and intelligence is actively developed from age 7 to $12 .{ }^{5,6}$ Body perception and postural control is matured from 7 to 12 years old. Riach and Hayes reported that the age of postural sway is $9-12$ years old. ${ }^{5}$ Cherng et al. ${ }^{6}$ suggested that the maturation of vision for standing balance at 7-10 years of age was the same as that of adults. During the young adolescent stage, postural control is trained by activity and experience. ${ }^{7}$

Own-body perception and postural control depends on the central nervous system to link sensory input from visual, somatosensory and vestibular systems with proper motor output. ${ }^{8}$ Own-body perception and experience is thought to be associated with the right hemisphere, and the right parietal lobe in particular. ${ }^{4}$ In addition, disturbance of the right hemisphere 
induces misperception of one's own body. ${ }^{9}$ Bauer et al. ${ }^{10}$ suggested that primary somatosensory areas (BA $3 \mathrm{a} / 3 \mathrm{~b}$ ) and left parieto-frontal areas play important roles in processing proprioceptive and own-body representation. Park et al. ${ }^{11}$ reported that the volume of vermis lobule VI-VII was correlated with static balance.

\section{Taekwondo and body perception}

Taekwondo is one of the most popular sports among children and adolescents in Korea. ${ }^{12}$ Taekwondo training in childhood is known to be effective in physical, cognitive, emotional and social development, including reduction of anxiety, encouraging independence and leadership, and controlling aggression. ${ }^{13}$ In addition, Taekwondo training can improve body perception and intelligence with postural control and sensory organization in healthy adolescents. ${ }^{14}$ The components of Taekwondo, including swift kicking, single leg standing, and fast action are thought to be related with balance control. ${ }^{15}$ Fong et al. ${ }^{16}$ reported that 3 months of Taekwondo training could improve standing balance in children with developmental coordination disorder. Vancampfort et al. ${ }^{17}$ reported that 6 months of physical activity could improve quality of life, perceived sports competence, physical fitness and perceived body attractiveness. In a study of the correlation between the central nervous system and peripheral performance effect, the actions (reaction time) of Taekwondo might be affected by brain function, which was stimulated by caffeine. ${ }^{18}$ However, few studies have investigated the correlation between body perception and brain function in Taekwondo training.

\section{Hypothesis}

In light of the increasing popularity of Taekwondo and as Taekwondo training is begun in the school age period, there is a need to examine the effects of Taekwondo training on body perception and brain development. This study aimed to assess body intelligence and brain connectivity in children with Taekwondo training as compared to children without Taekwondo training. In addition, we explored the correlation between body perception and brain connectivity in both groups.

\section{METHODS}

\section{Participants}

Fifteen children with Taekwondo training and 15 age- and sex-matched children who had no previous experience of Taekwondo training were recruited from the Department of Psychiatry, Chung Ang University Hospital, Seoul, Korea. Depression symptoms and symptoms of attention deficit/hyperactivity disorders $(\mathrm{ADHD})$ were evaluated with the Child Depressive Inventory $(\mathrm{CDI})^{19}$ and Dupaul's ADHD rating scale. ${ }^{20}$ In addi- tion, intelligence in all children was assessed with the KoreanWechsler Intelligence Scale for Children-IV (K-WISC-IV).

Exclusion criteria included: 1) psychiatric disorders, including depression and ADHD disorder, 2) history of head trauma with loss of consciousness, seizure disorder, multiple sclerosis, brain tumor, claustrophobia, and 3) IQ $<80$. The research protocol was approved by the Chung Ang University Hospital Institutional Review Board. Written informed assent was provided by adolescents and written informed consent was provided by parents.

\section{Body Intelligence Scale}

The Body Intelligence Scale (BIS) consists of 18 items with a 5-point Likert scale. It is composed of three subscales: the Energy Body Awareness (E-BAS), Comfort Body Awareness (C-BAS), and Inner Body Awareness subscales (I-BAS). ${ }^{2}$ EBAS measures awareness of energy on the inside and the exterior. Energy can be experienced through the five senses and body functions. C-BAS measures the feeling of comfort with one's body and the feeling of being at home. I-BAS measures awareness of minor changes inside of the body and changes in external circumstances. Cronbach's alphas for E-BAS, I-BAS, and C-BAS were $0.88,0.77$, and 0.82 , respectively. ${ }^{2}$ Cronbach's alphas of the Korean version of the E-BAS, I-BAS, and C-BAS were $0.73,0.74$, and 0.72 , respectively. ${ }^{21}$

\section{Brain analysis}

All magnetic resonance images were acquired using a 3.0 Tesla TX MRI scanner (Philips, Eindhoven, the Netherlands). Resting-state fMRI images were acquired with the following parameters: axially with an echo-planar imaging (EPI) sequence, $\mathrm{TR} / \mathrm{TE}=3000 / 40 \mathrm{~ms}$, 40 slices, $64 \times 64$ matrix, $90^{\circ}$ flip angle, 230-mm FOV, and 3-mm section thickness without a gap. For each participant, the scan of 230 volumes lasted $720 \mathrm{sec}-$ onds. Data were analyzed using the Data Processing Assistant for Resting-State fMRI (DPARSFA-http://www.restfmri.net), which is plug-in software that works with Statistical Parameter Mapping (SPM8, http://www.fil.ion.ucl.ac.uk/spm/software/spm8/) and the Resting-State fMRI Data Analysis toolkit (REST, http://resting-fmri.sourceforge.net). Images were corrected for slice acquisition time differences, realigned, normalized, spatially smoothed with a 4-mm FWHM kernel, detrended and temporally band-pass filtered to $0.01-0.08 \mathrm{~Hz}$. For the time series of each voxel, the first eigenvector of the time series in the white matter mask and the first eigenvector of the times series in the cerebrospinal fluid mask were regressed by using linear regression. To reduce physiological and other sources of noise and head motion-related covariates, an independent component-based noise correction method was used.

Amplitude of low-frequency fluctuations (ALFF) and func- 
tional connectivity (FC) were calculated from blood-oxygenation level dependent (BOLD) signal in the TKD and control groups. ALFF represents neural metabolic, hemodynamic, and neurophysiological status. ${ }^{22} \mathrm{FC}$ reflects signal synchronicity of low frequency fluctuation activity between different brain areas or brain network..$^{23} \mathrm{FC}$ analysis was applied to assess functional brain activity using REST software (seed regions approach). The protocol consisted of extracting the average BOLD time courses from a seed and computing the Pearson's correlation coefficients between this average time course and the BOLD time course of every other voxel. The correlation coefficients were converted to normally distributed z-scores using Fisher's z-transform. On first-level analysis of the comparisons between children with Taekwondo training and controls, ALFF analysis and functional connectivity maps with the cerebellum seed were formed using a two-sample t-test. For multiple comparisons with an extent of more than 40 contiguous voxels and participants to cluster analysis, the resulting maps were set to threshold using a $\mathrm{p}<0.05$ family-wise corrected type I error rate using Monte Carlo simulation (derived from an uncorrected $\mathrm{p}<0.001$ and 40 extended voxels). On second analysis, the correlation between BSI scores and eigenvalue of clusters which were found in first analysis was examined. Brain cell activity is allowed to flow around the network through synapses in brain. At each activation, a single unit of activity is transferred between a pair of neurons. During that transfer of activity, the spectrum of functional activity was represented by eigenvalue.

\section{RESULTS}

\section{Demographic characteristics and BIS}

There were no significant differences in age, education years, IQ, ARS-K scores, and CDI scores between children with Taekwondo training (TKD) and children without Taekwondo training (controls) (Table 1). However, the mean BIS score (77.1土
$4.9)$ in TKD was higher than that $(71.3 \pm 7.7)$ in controls. In addition, E-BAS score $(27.9 \pm 1.7)$ and I-BAS score $(26.1 \pm 1.7)$ in TKD were higher than those $(24.6 \pm 3.7 ; 22.6 \pm 3.1)$ in controls. The TKD group had 1,480 hours of Taekwondo training ( 2 hours/day $\times 5$ days/week $\times 37$ months) (Table 1 ).

\section{ALFF and FC analysis}

The TKD group showed increased ALFF in the right frontal precentral gyrus and right parietal precuneus, compared to the control group (Figure 1). The TKD group showed posi-

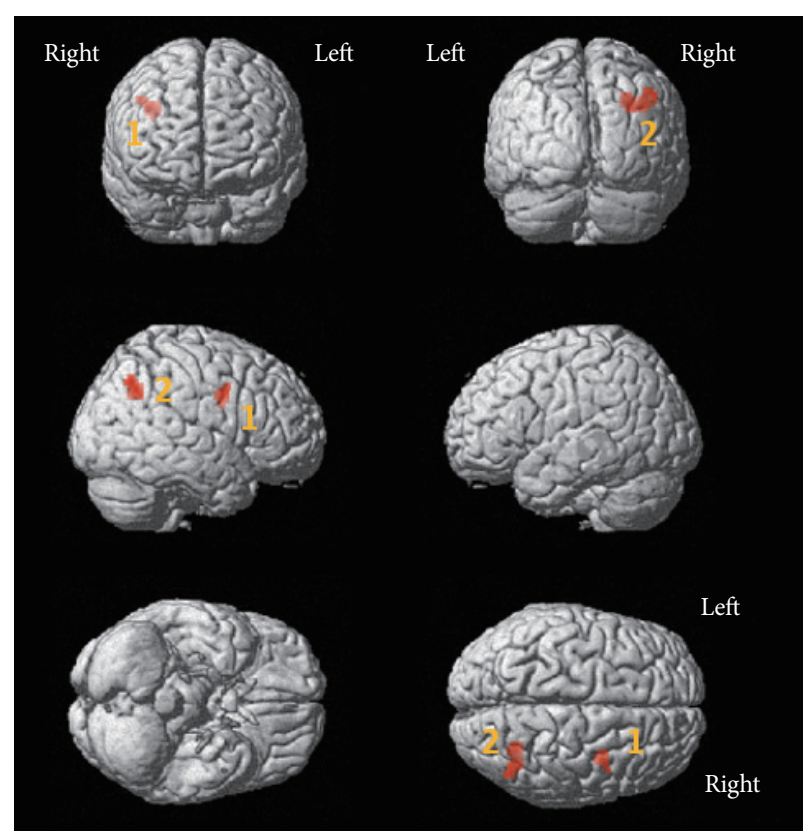

Figure 1. The comparison of amplitude of low-frequency fluctuations (ALFF) between children with taekwondo training and control groups. Red regions: increased ALFF in The TKD group, compared to the control group. 1: right frontal precentral gyrus (Broadmann 6), Talairach code $x, y, z=39,0,33$, clusters $(\kappa \epsilon)=41, T=4.49$, uncorrected $p<0.001,2$ : right parietal precuneus (Broadmann 19), Talairach code $x, y, z=30,-60,39$, clusters $(\kappa \in)=46, T=3.89$, uncorrected $p<0.001$.

Table 1. Demographic characteristics

\begin{tabular}{|c|c|c|c|}
\hline & Taekwondo $(\mathrm{N}=15)$ & Controls $(\mathrm{N}=13)$ & Statistics \\
\hline Sex (male/female) & $12 / 3$ & $10 / 5$ & $\chi^{2}=2.12, p=0.15$ \\
\hline Age & $12.6 \pm 0.9$ & $11.9 \pm 1.2$ & $\mathrm{z}=1.62, \mathrm{p}=0.11$ \\
\hline Education years & $5.6 \pm 0.9$ & $4.9 \pm 1.2$ & $\mathrm{z}=1.61, \mathrm{p}=0.11$ \\
\hline TKD training (months) & $37.1 \pm 9.7$ & - & - \\
\hline IQ & $99.0 \pm 10.1$ & $98.6 \pm 10.7$ & $\mathrm{z}=0.27, \mathrm{p}=0.79$ \\
\hline ARS-K & $4.4 \pm 3.1$ & $5.8 \pm 3.7$ & $\mathrm{z}=-1.16, \mathrm{p}=0.24$ \\
\hline CDI & $6.9 \pm 4.6$ & $5.9 \pm 3.9$ & $\mathrm{z}=0.39, \mathrm{p}=0.69$ \\
\hline BIS & $77.1 \pm 4.9$ & $71.3 \pm 7.7$ & $\mathrm{z}=2.33, \mathrm{p}=0.02$ \\
\hline E-BAS & $27.9 \pm 1.7$ & $24.6 \pm 3.7$ & $\mathrm{z}=2.87, \mathrm{p}<0.01$ \\
\hline C-BAS & $23.1 \pm 4.3$ & $24.1 \pm 4.4$ & $\mathrm{z}=-0.62, \mathrm{p}=0.53$ \\
\hline I-BAS & $26.1 \pm 1.7$ & $22.6 \pm 3.1$ & $\mathrm{z}=2.33, \mathrm{p}=0.01$ \\
\hline
\end{tabular}

ARS-K: Dupaul's ADHD Rating Scale, CDI: Children's Depression Inventory, BIS: Body Intelligence Scale, E-BAS: Energy Body Awareness, CBAS: Comfort Body Awareness, I-BAS: Inner Body Awareness 
tive cerebellum vermis (lobe VII) seed to the right frontal, left frontal and left parietal lobe. The control group showed positive cerebellum vermis (lobe VII) seed to the left frontal, parietal, and occipital cortex. Relative to the control group, the TKD group showed increased functional connectivity from cerebellum vermis (lobe VII) seed to the right inferior frontal gyrus (Figure 2, Table 2).

\section{Correlations between BIS and brain activity}

The brain activity (eigenvalue) of the precentral gyrus in all children was positively correlated with the mean total scores of BIS ( $r=0.53, p=0.03)$. In post hoc analysis, the brain activity of the precentral gyrus in TKD group was positively correlated with the mean total scores of BIS ( $r=0.71, p=0.03)$. Howev- er, the brain activity of the precentral gyrus in control group was not correlated with the mean total scores of BIS (Figure 3). There was no significant correlation between BSI scores and the eigenvalue of other clusters. The number of TKD training months was positively correlated with total BIS scores. However, there was no statistical significance $(\mathrm{r}=0.44, \mathrm{p}=0.11)$.

\section{DISCUSSION}

To the best of our knowledge, this is the first study to assess the effect of Taekwondo training on brain connectivity in children. The present study assessed body perception and brain function by measuring ALFF and cerebellum seed functional connectivity on resting-state functional MRI in children with

Table 2. Functional connectivity from cerebellum seed to other brain areas

\begin{tabular}{|c|c|c|c|c|c|c|}
\hline & \multicolumn{3}{|c|}{ Talairach code } & \multirow{2}{*}{$\mathrm{T}, \mathrm{P}_{\mathrm{FWE}<0.05}$} & \multirow{2}{*}{ Кє } & \multirow{2}{*}{ Regions } \\
\hline & $\mathrm{x}$ & $\mathrm{y}$ & $\mathrm{z}$ & & & \\
\hline \multirow[t]{5}{*}{ Taekwondo } & 6 & -60 & -36 & $13.36,<0.001$ & 745 & 1) Right cerebellum, cerebellar tonsil \\
\hline & -24 & 36 & 33 & $10.16,0.005$ & 109 & 2) Left middle frontal gyrus, BA 9 \\
\hline & 18 & 12 & 57 & $9.94,0.007$ & 108 & 3) Right middle frontal gyrus, BA 6 \\
\hline & 24 & 54 & 42 & $8.97,0.023$ & 100 & 4) Right superior frontal gyrus, BA 9 \\
\hline & -9 & -69 & 66 & $8.86,0.026$ & 100 & 5) Left parietal lobe, precuneus, BA 7 \\
\hline \multirow[t]{6}{*}{ Controls } & 24 & -63 & -33 & $16.04,<0.001$ & 239 & 1) Right cerebellum, cerebellar tonsil \\
\hline & -6 & -72 & 57 & $15.26,<0.001$ & 276 & 6) Left superior parietal lobule, BA 7 \\
\hline & -33 & 42 & 42 & $13.49,<0.001$ & 127 & 7) Left middle frontal gyrus, BA 8 \\
\hline & -42 & 3 & 54 & $12.21,0.001$ & 143 & 8) Left middle frontal gyrus, BA 6 \\
\hline & -3 & -96 & -15 & $11.87,0.001$ & 102 & 9) Left lingual gyrus, BA 18 \\
\hline & 0 & -87 & 18 & $10.71,0.003$ & 100 & 10) Left cuneus, BA 18 \\
\hline Group contrast & 39 & 33 & 6 & $\mathrm{~T}=4.88, \mathrm{P}_{\text {uncorrected }}<0.001$ & 115 & 11) Right inferior frontal gyrus, BA 46 \\
\hline
\end{tabular}

BA: Broadmann area, $\kappa \epsilon:$ clusters

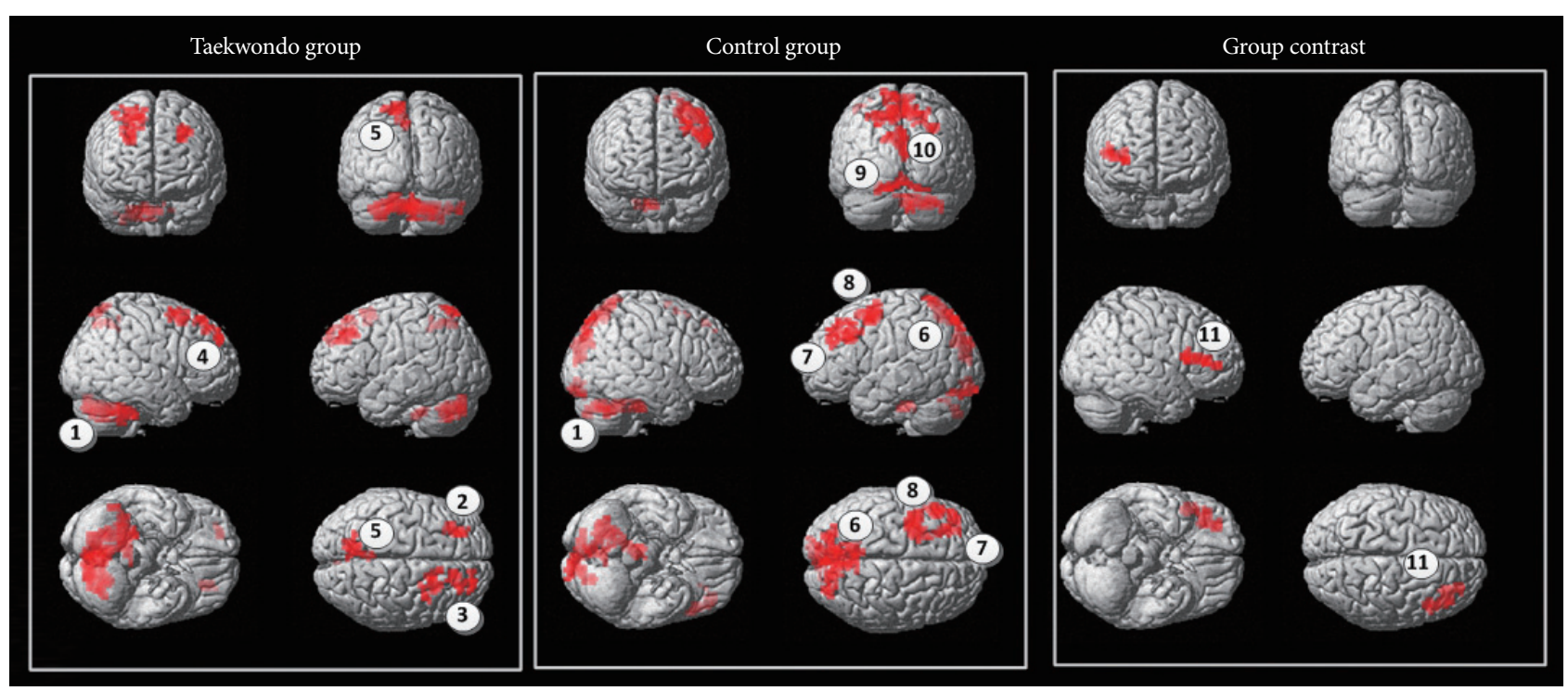

Figure 2. Functional connectivity from cerebellum seed to other brain areas. Red regions: increased functional connectivity from cerebellum vermis (lobe VII) seed to the right inferior frontal gyrus in Taekwondo group, compared to control group, see Table 2. 

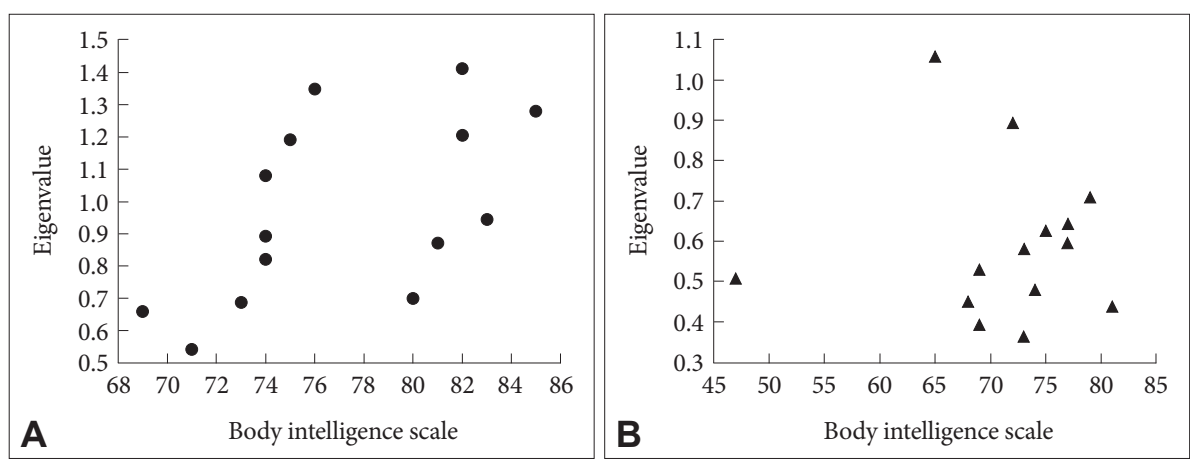

Figure 3. The correlation between brain activity within precentral gyrus and body intelligence scale. A: correlation between brain activity of the precentral gyrus in TKD group and the mean total scores of body intelligence scale (BIS) $(r=0.71, p=0.03)$. $\mathrm{B}$ : correlation between the brain activity of the precentral gyrus in control group and the mean total scores of BIS $(r=0.07, p=$ 0.74).
Taekwondo training. Relative to the control group, the TKD group showed increased body intelligence scores, increased ALFF value within the right precentral and precuneus areas, and increased connectivity between the cerebellum seed region to the right frontal cortex.

\section{BIS and Taekwondo}

The TKD group showed increased BIS scores, especially EBAS and I-BAS, compared to the control group. Those results indicate that TKD training improved the sense of body functioning in response to energy felt inside the physical body and exterior, as well as emotional control in response to the stress of inner body and external circumstances. ${ }^{2}$ TKD athletes may perceive themselves through complex body actions where imagination, fantasy, and the biological dimension of the body are intertwined. ${ }^{15,24}$ Taekwondo is composed of complex body actions. ${ }^{15}$ The performance in sport is a conflicting strategy (what action means in space) and the conflicting tactic of bodies-inspace. ${ }^{25}$ Vassiliki and Dimitra reported that TKD and Judo athletes had higher levels of emotional intelligence and healthier body image, compared to non-athlete controls. ${ }^{24}$ Hauser et al. ${ }^{26}$ reported that adolescents who accept their body have higher self-respect than those to hate their body, but on the contrary, a loss of physical organs and functions leads to inferior body image and inferior self-concept. Taekwondo training is known to encourage self-efficacy, confidence, and leadership cultivation, which studies have reported to be effective in improving learning efficiency. ${ }^{27,28}$ We speculate that improvement in selfconfidence and personal characteristics may be due to the body intelligence trained by Taekwondo body action. ${ }^{29}$ Future studies should directly assess the correlation between body intelligence and personal changes.

\section{Increased ALFF and FC}

The TKD group showed increased ALFF in the right frontal precentral gyrus and right parietal precuneus, compared to control groups. In addition, the brain activity of the precentral gyrus was positively correlated with BIS score in the current study. Physical exercise, including TKD training, may be adapted to brain network change. ${ }^{30,31}$ The precentral gyrus is known to be associated with the sense of space and time..$^{32}$ In addition, the precuneus plays a role in consciousness. ${ }^{33}$ With evidence that the pattern of theta activity within the precentral gyrus, precuneus, right temporoparietal junctions, and cerebellum in response to the experiences of time, space, and body perception, Berkovich-Ohana ${ }^{34}$ posited that the sense of time and space would be related to the body sensing process. Hodzic et al. ${ }^{35}$ suggested that the right superior and inferior parietal cortices were involved in the analysis of body-related information. ${ }^{36}$ In the current study, in the TKD group, the cerebellum was functionally connected to the right frontal cortex, while in the control group, the cerebellum was functionally connected to the left frontal and parietal cortices. The right hemisphere is known to be associated with the sense of space and body perception. ${ }^{4,9}$ In addition, the somatosensory network, including the parietal cortex and insular cortex, was associated with the feeling of limb ownership. ${ }^{30}$ The prefrontal cortex is known to be associated with self-referential processing ${ }^{37}$ and self-perception. ${ }^{38}$ Increased brain activity and functional connectivity may be due to continuous behavioral stimuli (complex Taekwondo actions)..$^{30}$ Taken together, we suggest that TKD training may improve activity within brain areas which are associated with body perception and self-control.

\section{Limitations}

There were several limitations to the current research. First, the small number of subjects was not sufficient to generalize the results. Second, the design of cross-sectional study is not enough showing the effect of Taekwondo training on brain connectivity. Finally, body perception and intelligence were assessed on a personal perception scale only. Future studies require a larger number of follow up cohort and assessment of body intelligence using physical methods.

\section{Conclusions}

Taekwondo training improved body intelligence and brain connectivity from the cerebellum to the parietal and frontal cortex. 


\section{Acknowledgments}

This work was supported by a grant of the Korean Health Technology R\& D Project, Ministry of Health \& Welfare, Republic of Korea (A120013) and grant of Korea Creative Content Agency (R2014040055).

\section{REFERENCES}

1. Fisher S. Development and Structure of Body Image. Hillsdale, NJ: Lawrence Erlbaum Associates, Inc; 1986.

2. Anderson BE. Management of forages for stocker cattle. Vet Clin North Am Food Anim Pract 2006;22:357-372.

3. Epstein J, Wiseman CV, Sunday SR, Klapper F, Alkalay L, Halmi KA. Neurocognitive evidence favors "top down" over "bottom up" mechanisms in the pathogenesis of body size distortions in anorexia nervosa. Eat Weight Disord 2001;6:140-147.

4. Giummarra MJ, Gibson SJ, Georgiou-Karistianis N, Bradshaw JL. Mechanisms underlying embodiment, disembodiment and loss of embodiment. Neurosci Biobehav Rev 2008;32:143-160.

5. Riach CL, Hayes KC. Maturation of postural sway in young children. Dev Med Child Neurol 1987;29:650-658.

6. Cherng RJ, Chen JJ, Su FC. Vestibular system in performance of standing balance of children and young adults under altered sensory conditions. Percept Mot Skills 2001;92:1167-1179.

7. Ionescu E, Morlet T, Froehlich P, Ferber-Viart C. Vestibular assessment with Balance Quest Normative data for children and young adults. Int J Pediatr Otorhinolaryngol 2006;70:1457-1465.

8. Nashner LM. Computerized Dynamic Posturography. In: Jacobson GP, Newman CW, Kartush JM, Editoris. Handbook of Balance Function and Testing. St. Louis: Mosby Year book, p.280-307, 1997.

9. Longo MR, Azanon E, Haggard P. More than skin deep: body representation beyond primary somatosensory cortex. Neuropsychologia 2010; 48:655-668.

10. Bauer CC, Diaz JL, Concha L, Barrios FA. Sustained attention to spontaneous thumb sensations activates brain somatosensory and other proprioceptive areas. Brain Cogn 2014;87:86-96.

11. Park IS, Yoon JH, Kim N, Rhyu IJ. Regional cerebellar volume reflects static balance in elite female short-track speed skaters. Int J Sports Med 2013;34:465-470.

12. Park YH, Gerrard J. Tae Kwon Do. The Ultimate Reference Guide to the World's Most Popular Marital Art. London: Ward Lock; 1989.

13. Kim SR, Seo JG. A study on value constitution factors of Taekwondo practices and practices satisfaction among elementary school students. Korean J Physical Edu 2004;43:581-591.

14. Fong SS, Fu SN, Ng GY. Taekwondo training speeds up the development of balance and sensory functions in young adolescents. J Sci Med Sport 2012;15:64-68.

15. Cromwell RL, Meyers PM, Meyers PE, Newton RA. Tae Kwon Do: an effective exercise for improving balance and walking ability in older adults. J Gerontol A Biol Sci Med Sci 2007;62:641-646.

16. Fong SS, Tsang WW, Ng GY. Taekwondo training improves sensory organization and balance control in children with developmental coordination disorder: a randomized controlled trial. Res Dev Disabil 2012;33: 85-95.

17. Vancampfort D, Probst M, Adriaens A, Pieters G, De Hert M, Stubbs B, et al. Changes in physical activity, physical fitness, self-perception and quality of life following a 6-month physical activity counseling and cognitive behavioral therapy program in outpatients with binge eating disorder. Psychiatry Res 2014;219:361-366.

18. Santos VG, Santos VR, Felippe LJ, Almeida JW Jr, Bertuzzi R, Kiss MA, et al. Caffeine reduces reaction time and improves performance in sim- ulated-contest of taekwondo. Nutrients 2014;6:637-649.

19. Cho SC, Lee YS. Development of the Korean form of the Kovacs' Children's Depression Inventory. J Korean Neuropsychiatr Assoc 1990;29:943955.

20. Dupaul GJ. Parent and teacher ratings of ADHD symptoms: psychometries in a community based sample. J Clin Child Psychol 1991;20:245253.

21. Park SG, Kim HM, Lee HS. A study on the application of a measuring instrument of the body insight: focusing on people with physical disabilities in sport for all. Korean Counc Phys Mult Health Disabil 2013;56:4158.

22. Fox MD, Raichle ME. Spontaneous fluctuations in brain activity observed with functional magnetic resonance imaging. Nat Rev Neurosci 2007;8:700-711.

23. Biswal B, Yetkin FZ, Haughton VM, Hyde JS. Functional connectivity in the motor cortex of resting human brain using echo-planar MRI. Magn Reson Med 1995;34:537-541.

24. Vassiliki C, Dimitra S. Emotional intelligence, body image and disordered eating attitudes in combat sport athletes. J Exerc Sci Fit 2009;7:104111.

25. Kreft L. Aesthetics of Football. Paper delivered at symposium "Football and Art". Preston: University of Preston and Museum of Football; 2010.

26. Hauser ST, Jacobson AM, Noam G, Powers S. Ego development and selfimage complexity in early adolescence. Longitudinal studies of psychiatric and diabetic patients. Arch Gen Psychiatry 1983;40:325-332.

27. Kurian M, Caterino LC, Kulhavy RW. Personality characteristics and duration of ATA Taekwondo training. Percept Mot Skills 1993;76:363-366.

28. Torabi MA, Khodayari A, Kohandel M. The relationship of leadership styles with achievement motivation and selfefficiency in Iran's Taekwondo elites. Eur J Exp Biol 2013;3:540-545.

29. Han DH, Park HW, Kee BS, Na C, Na DH, Zaichkowsky L. Performance enhancement with low stress and anxiety modulated by cognitive flexibility. Psychiatry Investig 2011;8:221-226.

30. van Dijk MT, van Wingen GA, van Lammeren A, Blom RM, de Kwaasteniet BP, Scholte HS, et al. Neural basis of limb ownership in individuals with body integrity identity disorder. PLoS One 2013;8:e72212.

31. Pons van Dijk G, Huijts M, Lodder J. Cognition improvement in Taekwondo novices over 40. Results from the SEKWONDO study. Front Aging Neurosci 2013;5:74.

32. Slagter HA, Lutz A, Greischar LL, Nieuwenhuis S, Davidson RJ. Theta phase synchrony and conscious target perception: impact of intensive mental training. J Cogn Neurosci 2009;21:1536-1549.

33. Cavanna AE, Trimble MR. The precuneus: a review of its functional anatomy and behavioural correlates. Brain 2006;129:564-583.

34. Berkovich-Ohana A, Dor-Ziderman Y, Glicksohn J, Goldstein A. Alterations in the sense of time, space, and body in the mindfulness-trained brain: a neurophenomenologically-guided MEG study. Front Psychol 2013;4:912.

35. Hodzic A, Muckli L, Singer W, Stirn A. Cortical responses to self and others. Hum Brain Mapp 2009;30:951-962.

36. Sim HB, Kang EH, Yu BH. Changes in cerebral cortex and limbic brain functions after short-term paroxetine treatment in panic disorder: an $[\mathrm{F}]$ FDG-PET pilot study. Psychiatry Investig 2010;7:215-219.

37. Mitchell JP, Banaji MR, Macrae CN. The link between social cognition and self-referential thought in the medial prefrontal cortex. J Cogn Neurosci 2005;17:1306-1315.

38. Waller G, Sines J, Meyer C, Mountford V. Body checking in the eating disorders: association with narcissistic characteristics. Eat Behav 2008;9: 163-169. 